\title{
First-line immunotherapy versus targeted therapy in patients with BRAF-mutant advanced melanoma: a real-world analysis
}

\author{
Anna C Pavlick*,1(i), Ruizhi Zhao², Cho-Han Lee², Corey Ritchings ${ }^{2}$ \& Sumati Rao² \\ ${ }^{1}$ Weill Cornell Medical Center, New York, NY 10021, USA \\ ${ }^{2}$ Bristol Myers Squibb, Princeton, NJ 08540, USA \\ *Author for correspondence: Tel.: +646962 6200; acp9008@med.cornell.edu
}

\begin{abstract}
Aim: To compare effectiveness of nivolumab + ipilimumab (NIVO + IPI) versus BRAF + MEK inhibitors (BRAFi + MEKi) in patients with BRAF-mutant advanced melanoma in the real-world setting. Materials \& methods: This study used the Flatiron Health electronic medical record database. Results: After adjusting for differences in baseline characteristics, NIVO + IPI was associated with a $32 \%$ reduction in risk of death versus BRAFi + MEKi. At a mean follow-up of $15-16$ months, $64 \%$ of NIVO + IPI patients and $43 \%$ of BRAFi + MEKi patients were alive; subsequent therapy was administered to 33 and $41 \%$ of patients, respectively. After first-line NIVO + IPI, $20 \%$ of patients died before subsequent therapy, whereas $32 \%$ died after first-line BRAFi + MEKi. Conclusion: In this real-world study, patients treated with first-line $\mathrm{NIVO}+$ IPI showed significant survival benefit versus those receiving first-line BRAFi + MEKi.
\end{abstract}

Lay abstract: Melanoma is an aggressive form of skin cancer. There are several treatment options for patients with advanced melanoma with a mutation in the BRAF gene including immunotherapy with nivolumab plus ipilimumab (NIVO + IPI) and targeted therapy with BRAF plus MEK inhibitors (BRAFi + MEKi). We aimed to compare these treatments in routine clinical practice. After analyzing data from the real-world Flatiron Health electronic medical record database, we found that the risk of death from advanced melanoma was $32 \%$ lower in patients who received NIVO + IPI compared with those who received BRAFi + MEKi. At a mean follow-up of 15-16 months, $64 \%$ of patients treated with NIVO + IPI were alive compared with $43 \%$ of patients treated with BRAFi + MEKi. Overall, 33 and $41 \%$ of patients received subsequent therapies after first-line treatment with NIVO + IPI or BRAFi + MEKi, respectively. After first-line treatment with NIVO + IPI, $20 \%$ of patients died before receiving subsequent therapy compared with $32 \%$ of patients who received first-line BRAFi + MEKi. In this real-world study, patients receiving first-line NIVO + IPI showed significant survival benefit versus those receiving first-line BRAFi + MEKi.

First draft submitted: 23 June 2020; Accepted for publication: 30 September 2020; Published online: 21 October 2020

Keywords: advanced melanoma $\bullet$ BRAF and MEK inhibitors $\bullet$ nivolumab plus ipilimumab • real-world study

Cutaneous melanoma is a common cancer in the USA, with an incidence that has risen consistently over the years to an estimated total of 96,480 new cases and 7230 associated deaths in 2019 [1]. Not long ago, patients with metastatic melanoma were known to have a poor prognosis, with a 5 -year survival rate of $<10 \%$ [2]. With the emergence of novel therapeutic approaches, treatment options for patients with advanced melanoma have evolved from chemotherapy and cytokine-based therapy to immunotherapy and targeted therapy in recent years [3]. Since 2011, several immune-based therapies that mediate checkpoint inhibition have been approved in the USA as monotherapies for the treatment of patients with advanced melanoma, including ipilimumab (anti-CTLA-4 antibody) and nivolumab and pembrolizumab (anti-PD-1 antibodies). Subsequently, nivolumab in combination with ipilimumab was approved in 2016 for the treatment of patients with advanced melanoma. Concurrently, a number of molecularly targeted therapies that are specific for the BRAF and MEK signaling pathways have also been approved for the treatment of advanced melanoma in patients whose tumors harbor a V600 mutation in the 


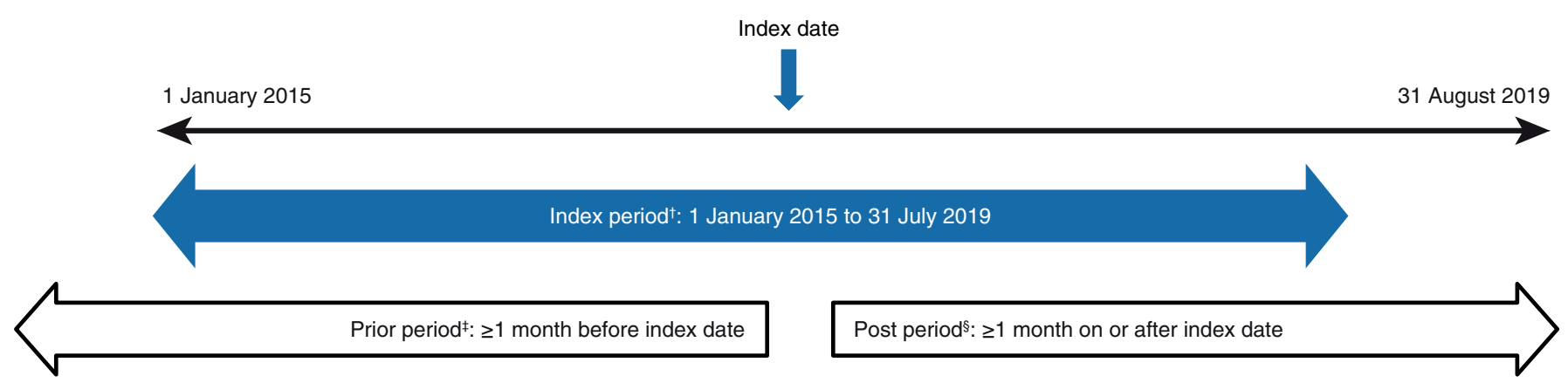

Figure 1. Study design.

${ }^{\dagger}$ Defined as the initial first-line nivolumab plus ipilimumab or BRAF plus MEK inhibitor prescription or administration date between 1 January 2015 and 31 July 2019.

¥Included $\geq 1$ month of patient medical data (records of any clinic visit or medication administration) prior to the index date.

$\S$ Included $\geq 1$ month of patient medical data (records of any clinic visit or medication administration) following and including the index date.

$B R A F$ gene [4]. These include the combination therapies dabrafenib plus trametinib, vemurafenib plus cobimetinib and encorafenib plus binimetinib.

Evidence from clinical trials has demonstrated improved overall survival (OS) and durable response with combination immunotherapy or targeted therapy in patients with BRAF-mutant advanced melanoma. In the Phase III CheckMate 067 trial (NCT01844505) [5], the combination of nivolumab and ipilimumab demonstrated durable OS with $60 \%$ of patients with $B R A F$-mutant advanced melanoma still alive at 5 years (the median OS had not been reached: 95\% CI: 50.7 months-not reached) [5]. Combination of BRAF and MEK inhibitors also showed promising OS in the Phase III COMBI-d [6] (NCT01584648), COMBI-v [7] (NCT01597908), coBRIM [8] (NCT01689519) and COLUMBUS (NCT01909453) [9] trials. In a pooled analysis of patients treated with dabrafenib plus trametinib in the COMBI-d and COMBI-v trials, the median OS was 25.9 months and $34 \%$ of patients were alive at 5 years [10]. A recent side-by-side analysis of data from the COMBI-v, coBRIM, and COLUMBUS trials showed that the median OS was numerically longer with encorafenib plus binimetinib (33.6 months) than with either dabrafenib plus trametinib (25.6 months) or vemurafenib plus cobimetinib (22.3 months) [11].

Despite the substantial benefit in survival that has been achieved with immunotherapy and targeted therapy in patients with $B R A F$-mutant advanced melanoma, a proportion of patients either do not respond to first-line treatment or develop resistance resulting in disease progression [12]. To address this, ongoing trials (DREAMseq [13] [NCT02224781] and SECOMBIT [14] [NCT02631447]) are investigating sequential dosing of nivolumab plus ipilimumab and BRAF plus MEK inhibitors to understand which treatment is more effective as first-line therapy. In the absence of data from these randomized trials, real-world experience can provide insights into optimal sequencing of immunotherapy and targeted therapy. In this study, we assessed baseline characteristics, clinical outcomes and treatment sequence patterns in patients with $B R A F$-mutant advanced melanoma who received first-line nivolumab plus ipilimumab or BRAF plus MEK inhibitor combination therapy in the real-world setting.

\section{Materials \& methods \\ Data source}

This retrospective observational study utilized the Flatiron Health oncology-focused electronic medical record (EMR) database, which contains data from more than 265 cancer clinics with 2500 healthcare practitioners across the USA. The patient-level data (de-identified) in the EMRs include both structured data (e.g., diagnosis codes, laboratory values, Eastern Cooperative Oncology Group [ECOG] performance status and prescribed drugs) and unstructured data collected via technology-enabled chart abstraction from physicians' notes (e.g., date of initial diagnosis, stage at initial diagnosis, biomarker testing including BRAF and PD-L1 status). Institutional review board approval of the study protocol was obtained prior to study conduct.

\section{Study design \& patients}

This study utilized data in the Flatiron Health EMR database dated between January 2015 and August 2019 (Figure 1). The index date was defined as the date of the first administration of nivolumab plus ipilimumab, 
dabrafenib plus trametinib, vemurafenib plus cobimetinib or encorafenib plus binimetinib. Patient medical data (records of clinic visits or medication administration) were collected $\geq 1$ month prior to the index date (prior period) and $\geq 1$ month after the index date (post period). Patients were followed until death, loss of follow-up or end of the study period (last observed visit date), whichever came first.

Eligible patients were aged 18 years or older, had a confirmed diagnosis of unresectable stage III or stage IV cutaneous melanoma, had two or more clinic visits occurring after January 2015, were treatment-naive and had received first-line nivolumab plus ipilimumab or BRAF plus MEK inhibitors on or after the initial diagnosis for advanced melanoma. Patients were excluded if they had $<1$ month of clinical activity during the prior period, had $<1$ month of clinical activity during the post period, were diagnosed with other primary cancers during the prior period or received $>1$ clinical trial therapy during the prior or post period. However, patients who died within 1 month of the index date were not excluded from the study.

\section{Clinical outcomes \& statistical analysis}

Descriptive analyses were used to describe demographics, baseline characteristics and treatment sequence patterns in the study population. OS was defined as the time from initiation of first-line therapy to the date of death. Progression-free survival (PFS) was defined as the time from initiation of first-line therapy until the date of first disease progression or death, whichever occurred first as documented by the physician. The Kaplan-Meier productlimit approach was used to estimate OS and PFS. Log-log transformation was used with Kaplan-Meier curves to estimate survival probabilities and 95\% CIs. The final model for OS was adjusted for first-line therapy, age and $\mathrm{LDH}$, which were significant predictors during stepwise model selection. Similarly, the final model for PFS was adjusted for first-line therapy, age, stage at initial diagnosis, distant recurrence and LDH. The index date was the start date for the survival analyses, and the event of interest was the occurrence of death or disease progression. Additional analyses for survival and death rates were estimated from patient disposition data.

\section{Results}

\section{Patient population}

A total of 1344 treatment-naive patients with advanced cutaneous melanoma who received first-line systemic therapy were identified in the Flatiron Health EMR database. Among these patients, 648 had $B R A F$-wild-type tumors, 557 had $B R A F$-mutant tumors and 139 had unknown $B R A F$ tumor status. Patients with $B R A F$-mutant melanoma were eligible and included in the analysis, among whom 107 (19\%) received first-line nivolumab plus ipilimumab, 227 (41\%) received BRAF plus MEK inhibitors and $223(40 \%)$ received other treatment as the index therapy. Other first-line treatments included nivolumab $(\mathrm{n}=98)$, pembrolizumab $(\mathrm{n}=93)$ and ipilimumab $(\mathrm{n}=32)$ monotherapies. In the BRAF plus MEK inhibitor group, 181 (80\%) patients received dabrafenib plus trametinib, $31(14 \%)$ received vemurafenib plus cobimetinib and $15(7 \%)$ received encorafenib plus binimetinib.

The mean age in the nivolumab plus ipilimumab and BRAF plus MEK inhibitor groups was 58 and 59 years, respectively; the majority of patients were male (65\% in both groups) and white (87 and $83 \%$ ) (Table 1). Differences between the groups were observed in the following clinical characteristics: the prevalence of comorbidities (66 vs $53 \%$, respectively; $\mathrm{p}=0.02$ ), ECOG performance status (50 vs 36\%; $\mathrm{p}=0.04)$ and LDH level (48 vs 33\%; $\mathrm{p}=0.04$ ). At the time of this analysis, the mean follow-up was 15.9 months (standard deviation: 13.4) in the nivolumab plus ipilimumab group and 15.0 months (standard deviation: 12.3) in the BRAF plus MEK inhibitor group $(\mathrm{p}=0.54)$.

\section{OS \& PFS}

Evaluation of OS and PFS demonstrated that a larger proportion of patients in the nivolumab plus ipilimumab group were alive over a longer follow-up period compared with those in the BRAF plus MEK inhibitor group. After adjusting for differences in demographics and baseline characteristics, nivolumab plus ipilimumab was associated with a $32 \%$ reduction in the risk of death compared with BRAF plus MEK inhibitors (hazard ratio [HR]: 0.68; 95\% CI: 0.47-0.98; $\mathrm{p}=0.038$ ). The median OS was 48.4 months (95\% CI: 21.4-48.4) in the nivolumab plus ipilimumab group and 17.7 months (95\% CI: 15.2-22.1) in the BRAF plus MEK inhibitor group; unadjusted OS probabilities were $69 \%$ at 1 year, $60 \%$ at 2 years and $58 \%$ at 3 years versus $61 \%$ at 1 year, $37 \%$ at 2 years and $27 \%$ at 3 years, respectively (Figure 2A). Similarly, after adjusting for differences in baseline characteristics, nivolumab plus ipilimumab was associated with a $29 \%$ reduction in the risk of progression or death compared with BRAF plus MEK inhibitors (HR: 0.71; 95\% CI: 0.52-0.95; $\mathrm{p}=0.022$ ). The median PFS was 7.8 months (95\% CI: 


\section{Table 1. Baseline and clinical characteristics.}

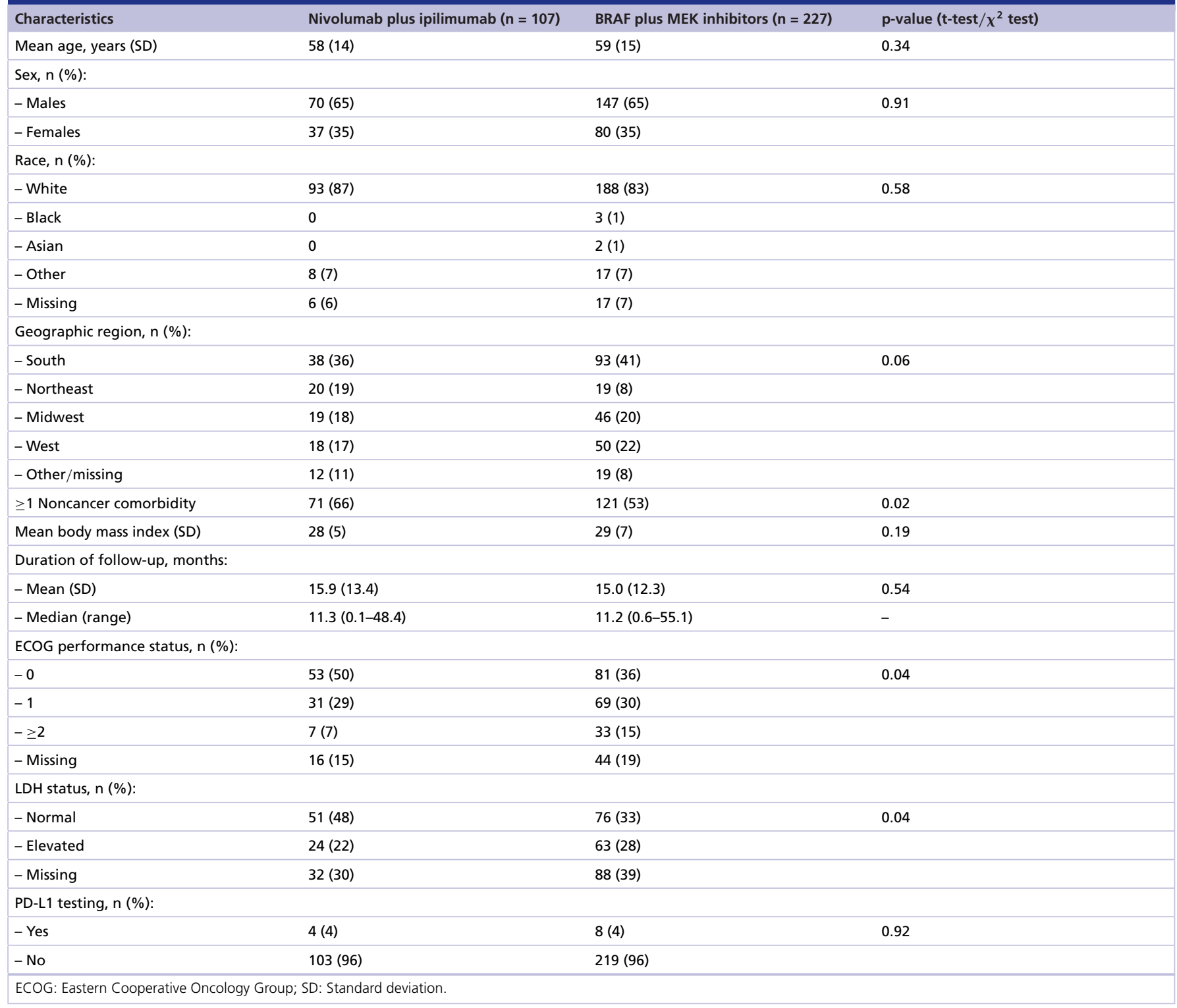

5.3-15.2) in the nivolumab plus ipilimumab group and 7.2 months (95\% CI: 5.6-8.8) in the BRAF plus MEK inhibitor group; unadjusted PFS probabilities were $42 \%$ at 1 year and $35 \%$ at 2 years versus $32 \%$ at 1 year and $14 \%$ at 2 years, respectively (Figure $2 \mathrm{~B}$ ).

\section{Treatment patterns}

Among patients who received first-line nivolumab plus ipilimumab, 33\% (35 of 107) received subsequent therapy; 23\% (25 of 107) received BRAF plus MEK inhibitors and 9\% (10 of 107) received other therapy (Figure 3A). Among those who received first-line BRAF + MEK inhibitors, 41\% (94 of 227) received subsequent therapy; 24\% (54 of 227) received anti-PD-1 monotherapy, 13\% (30 of 227) received nivolumab plus ipilimumab and 4\% (10 of 227) received other therapy (Figure 3B). Other subsequent therapies included ipilimumab monotherapy, chemotherapy, anti-PD-1 plus targeted therapy or therapy with nivolumab plus ipilimumab plus targeted therapy.

Survival \& death rates

Based on patient disposition data, 64\% (69 of 107) of patients were alive in the nivolumab plus ipilimumab group and 43\% (97 of 227) were alive in the BRAF plus MEK inhibitor group (Figure 4). Among the total population 
(A)

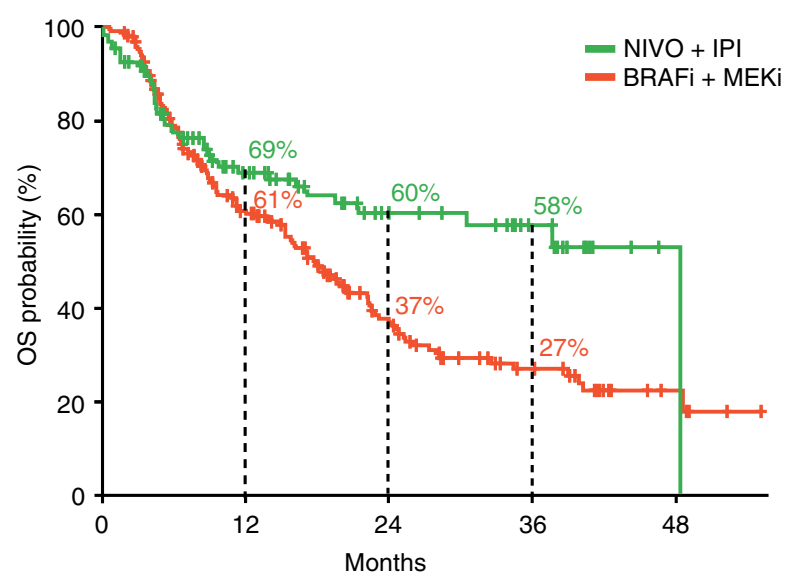

(B)

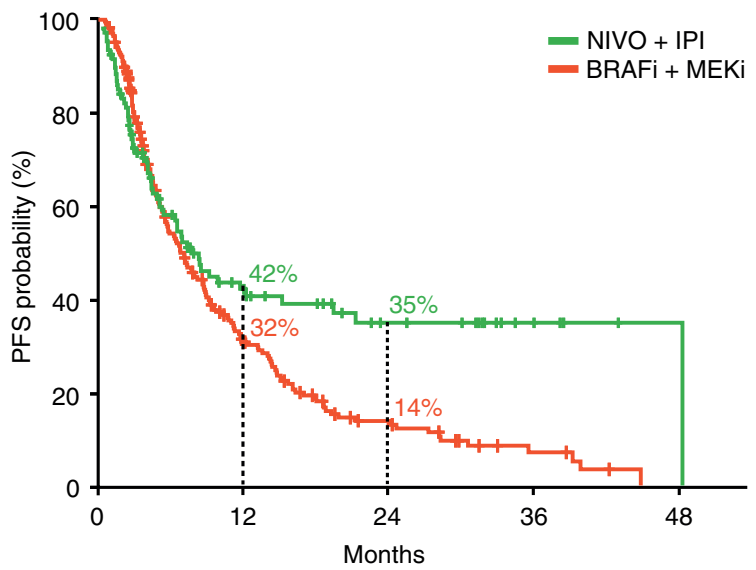

No. at risk

NIVO + IPI $107 \quad 30 \quad 15 \quad 351$

\begin{tabular}{|c|c|c|c|c|c|c|c|c|}
\hline No. at risk & & & & & No. at risk & & & \\
\hline NIVO + IPI 107 & 52 & 26 & 13 & 1 & NIVO + IPI 107 & 30 & 15 & 5 \\
\hline AFi + MEKi 227 & 109 & 46 & 21 & 5 & BRAFi + MEKi 227 & 54 & 18 & 5 \\
\hline
\end{tabular}

Figure 2. Kaplan-Meier survival curves. Unadjusted (A) OS and (B) PFS probabilities in patients with BRAF-mutant advanced melanoma treated with first-line nivolumab plus ipilimumab or BRAF plus MEK inhibitors.

BRAFi + MEKi: BRAF plus MEK inhibitors; NIVO + IPI: Nivolumab plus ipilimumab; OS: Overall survival; PFS: Progression-free survival.

(A)

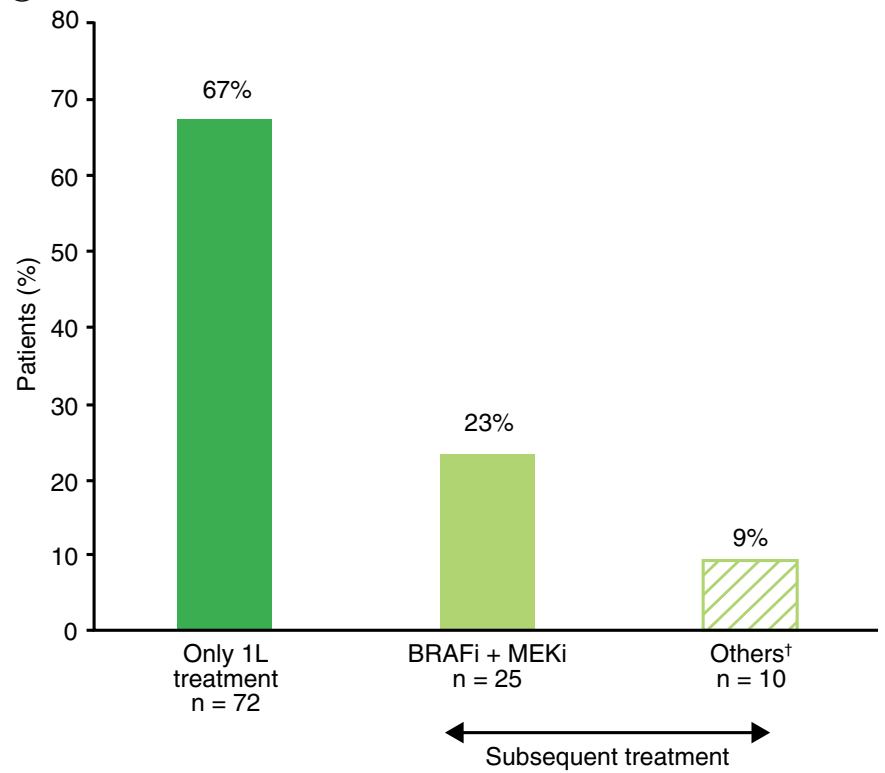

(B)

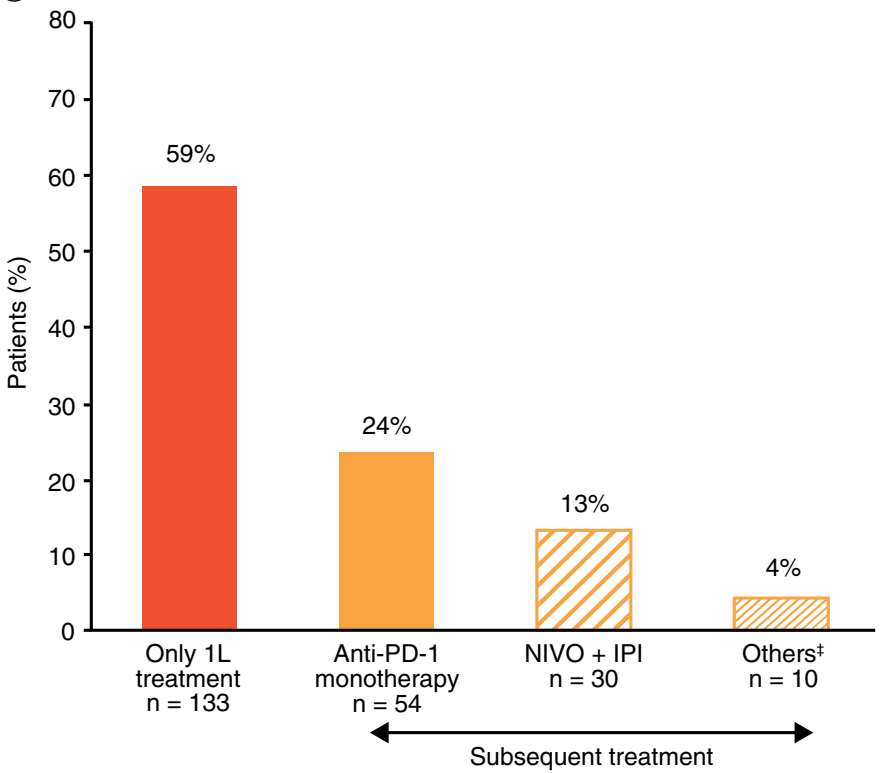

Figure 3. Treatment patterns. (A) Patients treated with first-line nivolumab plus ipilimumab. (B) Patients treated with first-line BRAF plus MEK inhibitors.

†Other subsequent therapies comprised anti-PD-1 monotherapy $(n=3)$, anti-PD-1 plus targeted therapy $(n=3)$, chemotherapy $(n=2)$, chemotherapy plus targeted therapy $(n=1)$ or nivolumab plus ipilimumab plus targeted therapy $(n=1)$.

¥Other subsequent therapies comprised anti-PD-1 plus targeted therapy $(n=7)$, ipilimumab monotherapy $(n=1)$, chemotherapy $(n=1)$ or anti-PD-1 combination ( $n=1)$.

1L: First-line; BRAFi + MEKi: BRAF plus MEK inhibitor; NIVO + IPI: Nivolumab plus ipilimumab.

treated with first-line nivolumab plus ipilimumab or BRAF plus MEK inhibitors, 20\% (21 of 107) and 32\% (73 of 227) of patients, respectively, died prior to receiving subsequent therapy.

Among patients who received only first-line treatment, $71 \%$ (51 of 72) and 45\% (60 of 133) were alive at the end of the study period in the nivolumab plus ipilimumab and BRAF plus MEK inhibitor groups, respectively (Figure 5). Among patients who received subsequent treatment, 51\% (18 of 35) and 39\% (37 of 94) were alive in 


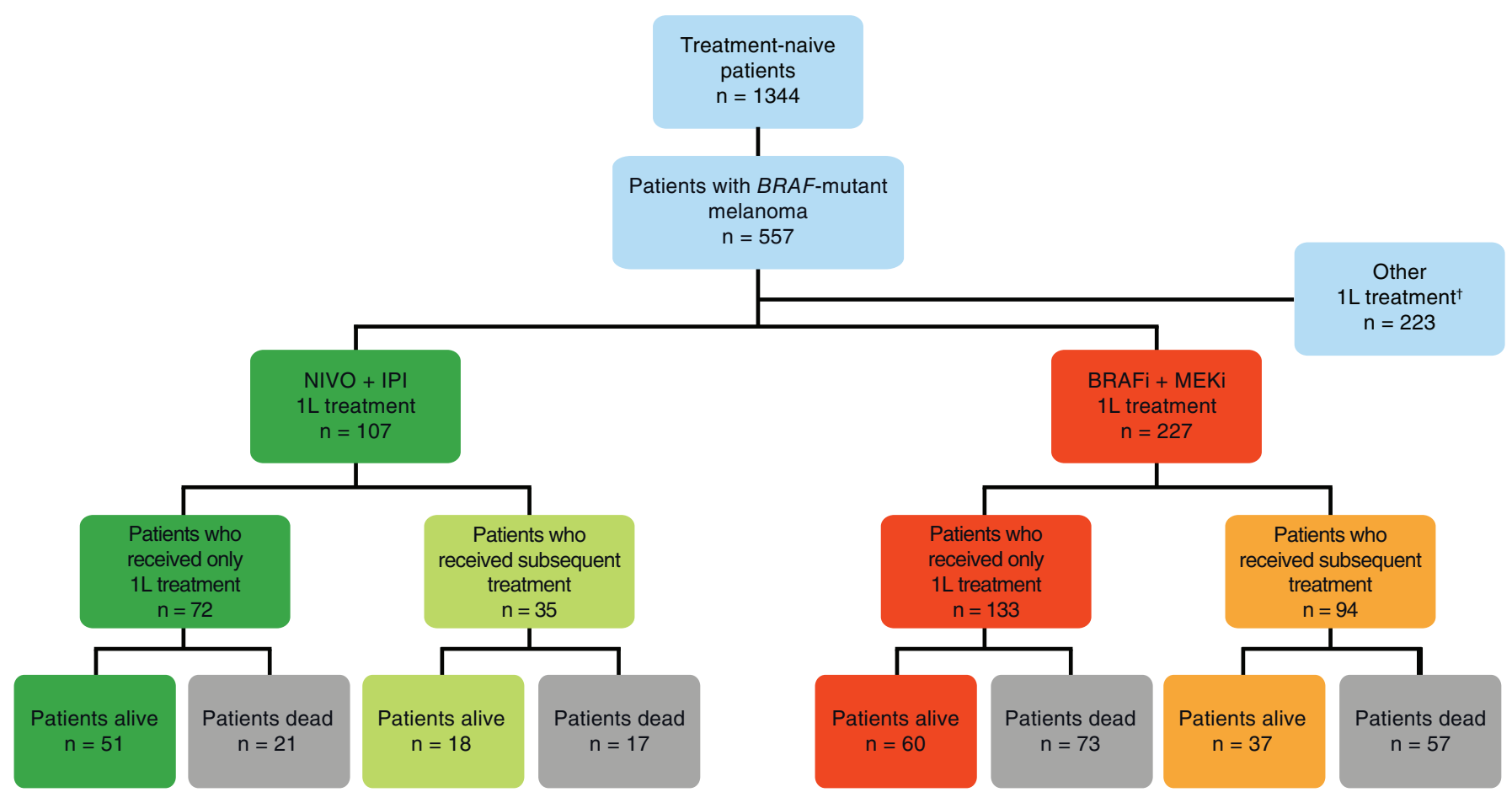

Figure 4. Patient disposition.

${ }^{\dagger}$ Nivolumab, pembrolizumab and ipilimumab monotherapies.

1L: First-line; BRAFi + MEKi: BRAF plus MEK inhibitors; NIVO + IPI: Nivolumab plus ipilimumab.

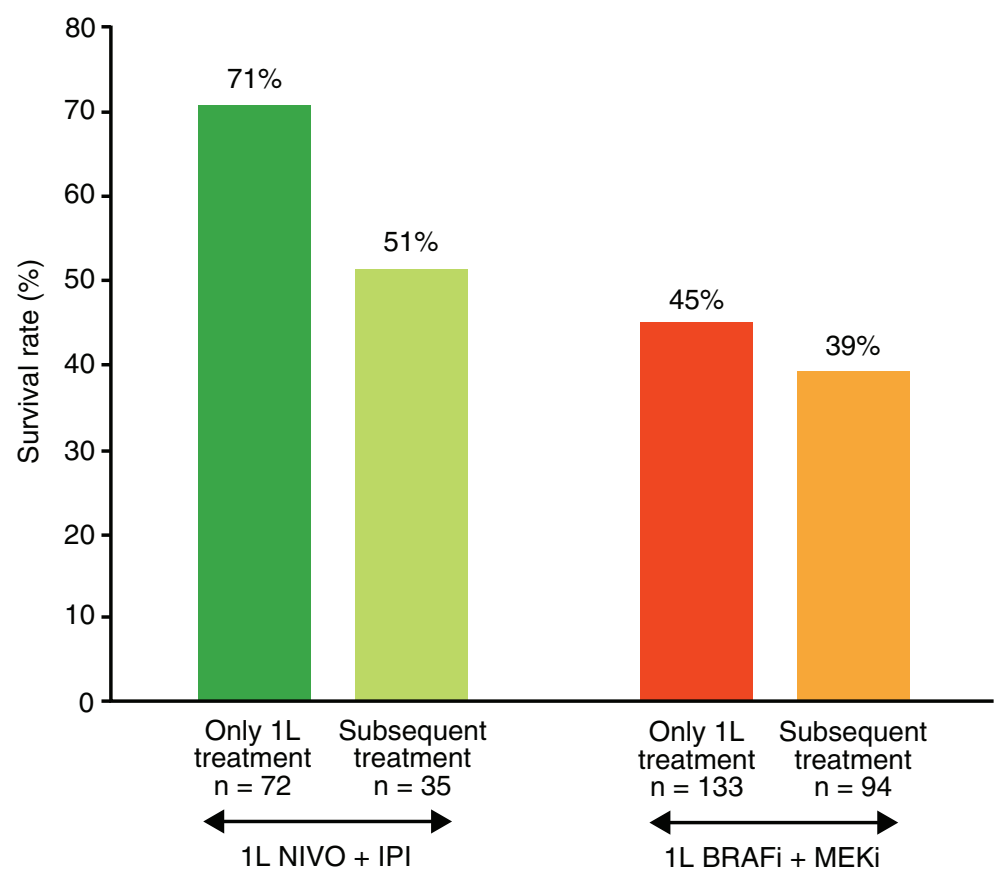

Figure 5. Survival rates in patients who received only first-line treatment and those who received subsequent treatment in the nivolumab plus ipilimumab and BRAF plus MEK inhibitor groups.

1L: First-line; BRAFi + MEKi: BRAF plus MEK inhibitor; NIVO + IPI: Nivolumab plus ipilimumab.

the nivolumab plus ipilimumab and BRAF plus MEK inhibitor groups, respectively.

In patients who received first-line nivolumab plus ipilimumab followed by BRAF plus MEK inhibitors or other therapy, 52\% (13 of 25) and 50\% (5 of 10) were alive, respectively (Figure 6A). In patients who received first-line BRAF plus MEK inhibitors followed by anti-PD-1 monotherapy, nivolumab plus ipilimumab or other therapy, $37 \%$ (20 of 54 ), $50 \%$ (15 of 30) and 20\% (2 of 10) were alive, respectively (Figure 6B). 
(A)

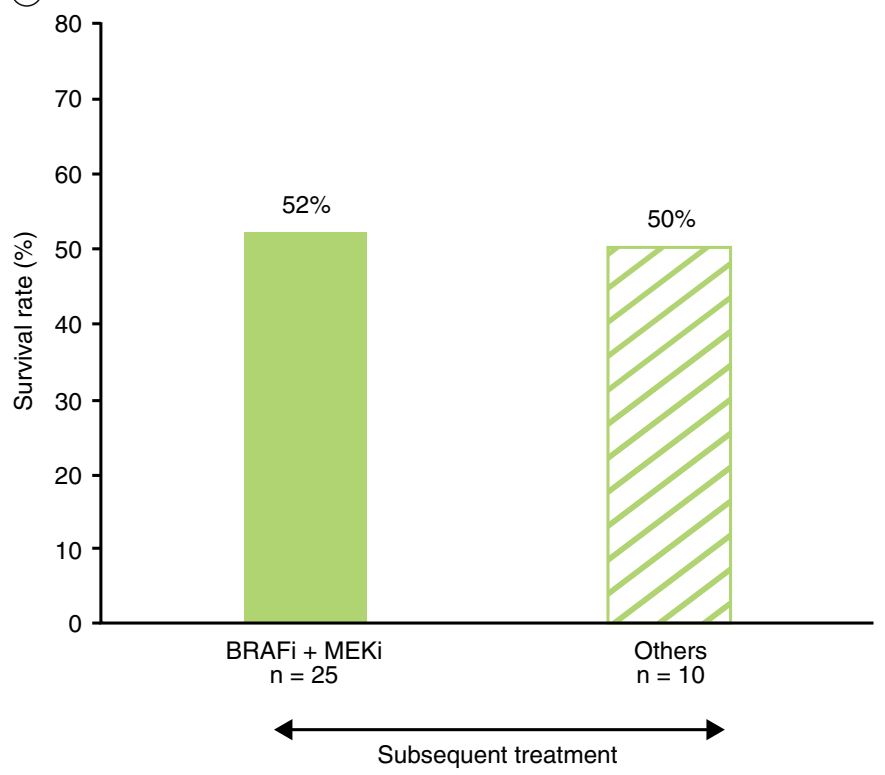

(B)

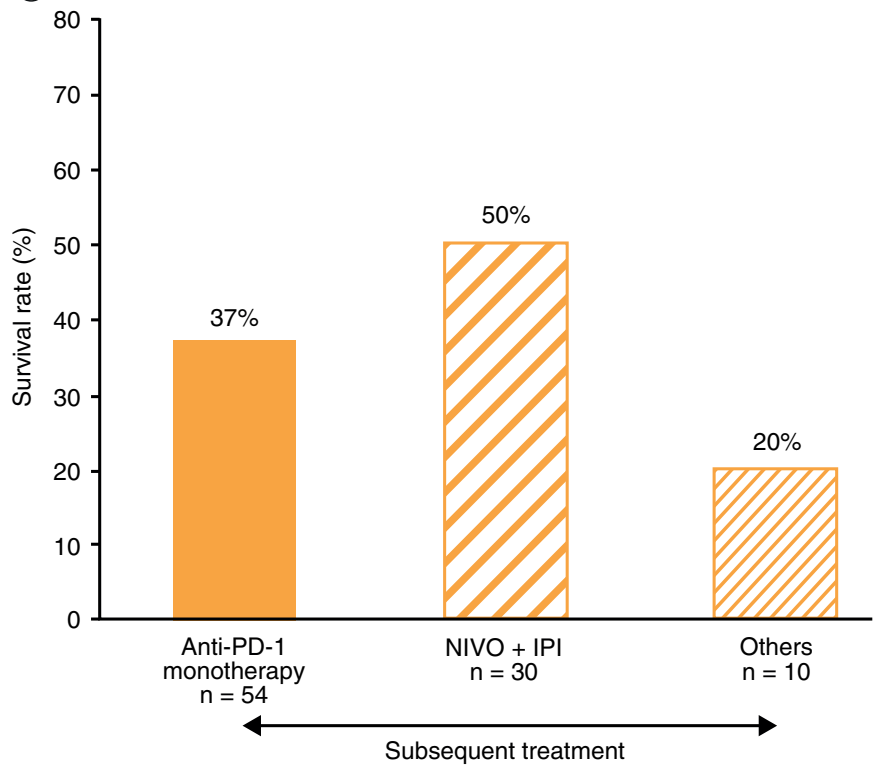

Figure 6. Survival rates in patients who received subsequent treatment. (A) Nivolumab plus ipilimumab group and (B) BRAF plus MEK inhibitor group.

BRAFi + MEKi: BRAF plus MEK inhibitor; NIVO + IPI: Nivolumab plus ipilimumab.

\section{Discussion}

In this real-world study population, patients with $B R A F$-mutant advanced melanoma who initiated treatment with first-line nivolumab plus ipilimumab showed significant OS and PFS benefits compared with those who initiated treatment with BRAF plus MEK inhibitors. More patients remained alive when treated with first-line nivolumab plus ipilimumab than with BRAF plus MEK inhibitors, whether they received only one line of treatment or multiple lines.

The patient population in this real-world study was similar to those reported in other real-world analyses [15,16]; it also included $12 \%$ of patients with ECOG performance status $\geq 2$, who are not typically enrolled in randomized clinical trials. In the present study, in the overall $B R A F$-mutant advanced melanoma population within the Flatiron database, a higher proportion of patients received BRAF plus MEK inhibitors (41\%) than nivolumab plus ipilimumab (19\%) as a first-line therapy, which may indicate a preference for targeted therapies among healthcare practitioners who treat patients with $B R A F$-mutant advanced melanoma.

The present analyses demonstrated that survival was improved in patients treated with nivolumab plus ipilimumab compared with BRAF plus MEK inhibitors based on two separate methods. In the first method, OS probability at 3 years estimated using Kaplan-Meier analysis was significantly higher with nivolumab plus ipilimumab than with BRAF plus MEK inhibitors (58 vs $27 \%$ ), with an adjusted HR demonstrating a $32 \%$ reduced risk of death. In addition, PFS with nivolumab plus ipilimumab treatment was significantly longer than that with BRAF plus MEK inhibitors, with an unadjusted PFS probability of 35 versus $14 \%$ at 2 years and an adjusted HR demonstrating a $29 \%$ reduced risk of progression. In the second method, descriptive analysis of the medical records based on patient disposition data included in this study showed that more patients were alive on first-line nivolumab plus ipilimumab than on BRAF plus MEK inhibitors (64 vs 43\%) at the end of the study period, with similar follow-up times in each arm. This was seen whether or not subsequent therapy was initiated.

Survival results obtained in this study were comparable with those observed in other real-world studies, clinical trials and indirect cross-trial analyses. A recent real-world retrospective analysis utilizing the Flatiron database also showed that treatment with first-line nivolumab plus ipilimumab or anti-PD-1 therapy prolonged survival compared with treatment with BRAF plus MEK inhibitors in patients with $B R A F$-mutant advanced melanoma [17]. However, treatment patterns and survival in patients who received subsequent therapy were not investigated. In randomized clinical trials, 3-year OS rates were $68 \%$ in patients with $B R A F$-mutant melanoma treated with nivolumab plus ipilimumab (CheckMate 067) [18] and 44\% in those treated with dabrafenib plus trametinib (COMBI-d) [6], 
while PFS rates were 39 and 22\%, respectively. The estimated survival probabilities in the current study were somewhat lower than those observed in clinical trials, which may have resulted from the inclusion of patients with an ECOG performance status $\geq 2$. Finally, a matching-adjusted indirect comparison of the CheckMate 067 and 069 and COMBI-d/v, coBRIM and COLUMBUS trials showed that patients who received first-line nivolumab plus ipilimumab demonstrated improved OS and PFS compared with those who received BRAF plus MEK inhibitors $[19,20]$.

The current study provides important information about patterns of treatment sequences in this real-world patient population and outcomes achieved from each sequence. Subsequent systemic therapy was administered to fewer patients in the nivolumab plus ipilimumab group than in the BRAF plus MEK inhibitor group (33 vs $41 \%)$. One possible reason for the higher use of subsequent therapy in those in the BRAF plus MEK inhibitor group is the development of acquired resistance to treatment in these patients. This treatment resistance may have been reflected in the shorter median duration of response in patients with BRAF-mutant tumors treated with BRAF plus MEK inhibitors (12-19 months) [6,8,9] than in those treated with nivolumab plus ipilimumab (not reached) [5] in randomized clinical trials. Overall, 20 and $32 \%$ of patients died in the nivolumab plus ipilimumab and BRAF plus MEK inhibitor groups, respectively, without receiving subsequent therapy. These results indicate that while melanoma patients with $B R A F$-mutant tumors have multiple classes of medications available to them that can be sequenced, the choice of first-line treatment is important, as this may be the only treatment they have an opportunity to receive. Whether patients received only one line of treatment or more, a higher proportion of those who received first-line nivolumab plus ipilimumab were alive at the end of the treatment period than those who received BRAF plus MEK inhibitors.

The optimal sequencing of treatment with immunotherapy and targeted therapy in patients with $B R A F$-mutant advanced melanoma has been a topic of considerable interest [12]. As the results of ongoing clinical trials investigating the optimal sequencing of these combinations are not yet available [13,14], cross-trial comparisons and retrospective analyses can be used to assess the relative benefits of these treatments. In the current study, survival probabilities were similar between patients treated with first-line nivolumab plus ipilimumab followed by subsequent BRAF plus MEK inhibitors and those treated with first-line BRAF plus MEK inhibitors followed by subsequent nivolumab plus ipilimumab (52 vs $50 \%$ ). However, patients who received anti-PD-1 monotherapy subsequent to BRAF plus MEK inhibitors had a numerically lower survival rate than those who received nivolumab plus ipilimumab as subsequent therapy (37 vs 50\%). Although the survival rate was better with subsequent nivolumab plus ipilimumab, a higher proportion of patients received subsequent anti-PD-1 monotherapy than nivolumab plus ipilimumab (24 vs $13 \%$ ), which may have been due to the better safety profile with anti-PD-1 monotherapy. A sequencing model incorporating data from the CheckMate 067 and 069 and COMBI-v and COMBI-d studies also showed that total life-years were higher in patients who initiated first-line nivolumab plus ipilimumab followed by subsequent BRAF plus MEK inhibitors (8.4 years) compared with first-line anti-PD-1 therapies followed by subsequent BRAF plus MEK inhibitors (6.9 years) or first-line BRAF plus MEK inhibitors followed by subsequent anti-PD-1 therapy (3.2 years) [21]. Although this study did not include patients treated with first-line BRAF plus MEK inhibitors followed by nivolumab plus ipilimumab due to lack of data availability on this sequence, the results showed that treatment with first-line nivolumab plus ipilimumab followed by subsequent BRAF plus MEK inhibitors yielded the most benefit and was cost-effective [21]. In contrast to the results of the current study, a recent retrospective study showed that survival was similar in patients receiving the combination of nivolumab plus ipilimumab or anti-PD-1 monotherapies following failure of targeted therapy in those with poor prognostic factors [22]. The reason for the different outcomes in these two studies may, in part, be attributed to differences in the patient populations.

The limitations of this analysis were largely inherent to those of a retrospective observational study and should be considered in interpreting these findings. Data were limited in a number of clinical characteristic categories: For example, ECOG performance status and LDH status were missing in 18 and 36\% of patients, respectively; M stage at advanced melanoma diagnosis was not available, and most patients were not tested for PD-L1 status. Due to the small sample size, baseline characteristics were not matched between the treatment groups; however, we adjusted for baseline differences in the survival analyses. Furthermore, adverse events associated with immunotherapy and targeted therapy treatment were not available in this database. The adverse event profile is an important consideration in the overall benefit-risk discussion with patients. In addition, the follow-up period was relatively short in this study, and therefore long-term effects were not captured. Previous analyses have suggested that the benefit of nivolumab plus ipilimumab may increase over time [19,20]. 


\section{Conclusion}

To our knowledge, no clinical trials have reported results that have directly compared the effectiveness of combination therapy with nivolumab plus ipilimumab versus BRAF plus MEK inhibitors in patients with $B R A F$-mutant advanced melanoma. In the absence of head-to-head data, a real-world study such as the one presented here may help guide treatment decisions. The results of this observational study demonstrated that patients with advanced melanoma initiating treatment with nivolumab plus ipilimumab had a 32\% reduced risk of death compared with those initiating treatment with BRAF plus MEK inhibitors, despite having a lower proportion of patients who received subsequent therapies. These results will require further validation in ongoing randomized trials that directly compare these combination approaches.

\section{Summary points}

- Nivolumab plus ipilimumab and several BRAF plus MEK inhibitors are approved treatments for patients with BRAF-mutant advanced melanoma; however, no head-to-head clinical trial data exist.

- Using Flatiron electronic medical records, we compared the effectiveness of nivolumab plus ipilimumab versus BRAF plus MEK inhibitors in treatment-naive patients with BRAF-mutant advanced melanoma.

- After adjusting for differences in baseline characteristics, nivolumab plus ipilimumab was associated with a $32 \%$ reduction in the risk of death compared with BRAF plus MEK inhibitors (hazard ratio: $0.68 ; 95 \% \mathrm{Cl}: 0.47-0.98$; $p=0.038)$.

- At a mean follow-up of $15-16$ months, $64 \%$ of patients in the nivolumab plus ipilimumab group were alive, whereas $43 \%$ were alive in the BRAF plus MEK inhibitor group.

- A total of $20 \%$ of patients in the nivolumab plus ipilimumab group died prior to subsequent therapy, whereas $32 \%$ died in the BRAF plus MEK inhibitor group; 33 and $41 \%$ of patients received subsequent therapies, respectively.

- In this real-world study, patients with BRAF-mutant advanced melanoma treated with first-line nivolumab plus ipilimumab showed significant survival benefit compared with those treated with first-line BRAF plus MEK inhibitors.

\section{Author contributions}

AC Pavlick participated in study conception, design, and data interpretation; R Zhao and C-H Lee participated in data acquisition, analysis and interpretation; C Ritchings and S Rao participated in study conception, design and data interpretation. All authors reviewed the manuscript and provided approval for submission.

\section{Acknowledgments}

The authors would like to acknowledge Bristol Myers Squibb (NJ, USA) and ONO Pharmaceutical Company Ltd (Osaka, Japan).

Financial \& competing interests disclosure

AC Pavlick has received personal fees and served on the advisory board for Bristol Myers Squibb, Regeneron, and Sanofi. R Zhao, C-H Lee, C Ritchings, and S Rao are employees of Bristol Myers Squibb. This study was supported by Bristol Myers Squibb (NJ, USA). The authors have no other relevant affiliations or financial involvement with any organization or entity with a financial interest in or financial conflict with the subject matter or materials discussed in the manuscript apart from those disclosed.

Writing and editorial assistance were provided by K Parai and A Lockett of Ashfield Healthcare Communications, funded by Bristol Myers Squibb.

\section{Ethical conduct of research}

Institutional review board approval of the study protocol was obtained prior to study conduct.

\section{Availability of data}

The Bristol Myers Squibb policy on data sharing may be found at www.bms.com/researchers-and-partners/independent-research/ data-sharing-request-process.html.

\section{Open access}

This work is licensed under the Attribution-NonCommercial-NoDerivatives 4.0 Unported License. To view a copy of this license, visit http://creativecommons.org/licenses/by-nc-nd/4.0/ 


\section{References}

Papers of special note have been highlighted as: $\bullet$ of interest; $\bullet \bullet$ of considerable interest

1. National Cancer Institute, Surveillance, Epidemiology, and End Results Program. Cancer stat facts: melanoma of the skin (2019). https://seer.cancer.gov/statfacts/html/melan.html

2. Garbe C, Eigentler TK, Keilholz U, Hauschild A, Kirkwood JM. Systematic review of medical treatment in melanoma: current status and future prospects. Oncologist 16(1), 5-24 (2011).

3. Furue M, Ito T, Wada N, Wada M, Kadono T, Uchi H. Melanoma and immune checkpoint inhibitors. Curr. Oncol. Rep. $20(3), 29$ (2018).

4. Vanella V, Festino L, Trojaniello C et al. The role of BRAF-targeted therapy for advanced melanoma in the immunotherapy era. Curr. Oncol. Rep. 21(9), 76 (2019).

5. Larkin J, Chiarion-Sileni V, Gonzalez R et al. Five-year survival with combined nivolumab and ipilimumab in advanced melanoma. $N$. Engl. J. Med. 381(16), 1535-1546 (2019).

-• The Phase III CheckMate 067 study in patients with advanced melanoma demonstrates sustained long-term overall survival at 5 years in a greater percentage of patients with nivolumab plus ipilimumab or nivolumab alone than in those with ipilimumab alone.

6. Long GV, Flaherty KT, Stroyakovskiy D et al. Dabrafenib plus trametinib versus dabrafenib monotherapy in patients with metastatic BRAF V600E/K-mutant melanoma: long-term survival and safety analysis of a Phase III study. Ann. Oncol. 28(7), 1631-1639 (2017).

7. Robert C, Karaszewska B, Schachter J et al. Two year estimate of overall survival in COMBI-v, a randomized, open-label, Phase III study comparing the combination of dabrafenib (D) and trametinib (T) with vemurafenib (Vem) as first-line therapy in patients (pts) with unresectable or metastatic BRAF V600E/K mutation-positive cutaneous melanoma. Eur. J. Cancer 51(Suppl. 3), S663, Abstract 3301 (2015).

8. Ascierto PA, McArthur GA, Dréno B et al. Cobimetinib combined with vemurafenib in advanced BRAFV600-mutant melanoma (coBRIM): updated efficacy results from a randomised, double-blind, Phase III trial. Lancet Oncol. 17(9), 1248-1260 (2016).

9. Dummer R, Ascierto PA, Gogas HJ et al. Overall survival in patients with BRAF-mutant melanoma receiving encorafenib plus binimetinib versus vemurafenib or encorafenib (COLUMBUS): a multicentre, open-label, randomised, Phase III trial. Lancet Oncol. 19(10), 1315-1327 (2018).

10. Robert C, Grob JJ, Stroyakovskiy D et al. Five-year outcomes with dabrafenib plus trametinib in metastatic melanoma. N. Engl. J. Med. 381(7), 626-636 (2019).

-. Pooled data from two Phase III trials shows that first-line treatment with dabrafenib plus trametinib led to long-term benefit in approximately one-third of the patients who had advanced melanoma with a BRAF mutation.

11. Hamid O, Cowey CL, Offner M et al. Efficacy, safety, and tolerability of approved combination BRAF and MEK inhibitor regimens for BRAF-mutant melanoma. Cancers (Basel) 11(11), 1642 (2019).

12. Pavlick AC, Fecher L, Ascierto PA et al. Frontline therapy for BRAF-mutated metastatic melanoma: how do you choose, and is there one correct answer? Am. Soc. Clin. Oncol. Educ. Book. 39, 564-571 (2019). https://pubmed.ncbi.nlm.nih.gov/31099689/

13. ClinicalTrials.gov. Dabrafenib and trametinib followed by ipilimumab and nivolumab or ipilimumab and nivolumab followed by dabrafenib and trametinib in treating patients with stage III-IV BRAFV600 melanoma (2019).

http://www.clinicaltrials.gov/ct2/show/NCT02224781

14. ClinicalTrials.gov. Sequential combo immune and target therapy study (SECOMBIT) (2019). https://clinicaltrials.gov/ct2/show/NCT02631447

15. Liu FX, Ou W, Diede SJ, Whitman ED. Real-world experience with pembrolizumab in patients with advanced melanoma: a large retrospective observational study. Medicine (Baltimore) 98(30), e16542 (2019).

16. Cowey CL, Liu FX, Boyd M, Aguilar KM, Krepler C. Real-world treatment patterns and clinical outcomes among patients with advanced melanoma: a retrospective, community oncology-based cohort study (a STROBE-compliant article). Medicine (Baltimore) 98(28), e16328 (2019).

17. Moser JC, Chen D, Hu-Lieskovan S et al. Real-world survival of patients with advanced BRAF V600 mutated melanoma treated with front-line BRAF/MEK inhibitors, anti-PD-1 antibodies, or nivolumab/ipilimumab. Cancer Med. 8(18), 7637-7643 (2019).

-• This real-world study shows that patients with BRAF mutant advanced melanoma treated with front-line nivolumab plus ipilimumab or anti-PD1 agents had longer survival compared to those treated with front-line BRAF plus MEK inhibitors.

18. Wolchok JD, Chiarion-Sileni V, Gonzalez R et al. Overall survival with combined nivolumab and ipilimumab in advanced melanoma. N. Engl. J. Med. 377(14), 1345-1356 (2017).

19. Atkins MB, Tarhini A, Rael M et al. Comparative efficacy of combination immunotherapy and targeted therapy in the treatment of BRAF-mutant advanced melanoma: a matching-adjusted indirect comparison. Immunotherapy. 11(7), 617-629 (2019).

- After adjusting for differences in patient population, this study showed that nivolumab plus ipilimumab demonstrated a significant survival benefit over BRAF plus MEK inhibitors starting at 12 months and beyond. 
20. Tarhini AA, McDermott DF, Wang R et al. Long-term survival benefit of nivolumab plus ipilimumab versus BRAF + MEK inhibitors for patients with BRAF-mutant advanced melanoma. Presented at: 16th International Congress of the Society for Melanoma Research. UT, USA (20-23 November 2019).

21. Tarhini A, McDermott D, Ambavane A et al. Clinical and economic outcomes associated with treatment sequences in patients with BRAF-mutant advanced melanoma. Immunotherapy 11(4), 283-295 (2019).

- Findings from this study showed that initiating first-line treatment with an immune-oncology agent provided a longer survival benefit compared with initiating treatment with a BRAF plus MEK inhibitor combination.

22. Kreft S, Gesierich A, Eigentler T et al. Efficacy of PD-1-based immunotherapy after radiologic progression on targeted therapy in stage IV melanoma. Eur. J. Cancer 116, 207-215 (2019).

- Demonstrates that programmed death 1 monotherapy and combined nivolumab plus ipilimumab showed similar activity and outcome in patients with melanoma resistant to BRAF plus MEK inhibition. 